\title{
Density structure of dense cores in the Cepheus molecular cloud
}

\author{
K. Sunada ${ }^{1}$, T. Hasegawa ${ }^{2}$, M. Hayashi $^{1}$, Y. Fukul ${ }^{3}$, and K. Sugitani ${ }^{4}$ \\ 1. Department of Astronomy, University of Tokyo \\ 2. Instilute of Astronomy, Universily of Tokyo \\ 3. Deparlmenl of Astrophysics, Nagoya Universily \\ 4. College of General Education, Nagoya Cily Universily
}

SUMMARY We mapped 5. dense cores in the Cepheus molecular cloud in the optically thin $\mathrm{C}^{18} \mathrm{O}(J=1-0)$ line using the $45-\mathrm{m}$ telescope at Nobeyama and derived density profiles around those cores assuming spherical symmetry. Cloud cores are selected from the ${ }^{13} \mathrm{CO}(J=1-0)$ map obtained through the unbiased survey program with the 4-m telescope at Nagoya university.

The results are summarized as below:

1. The cores with protostellar IRAS sources have the density profile consistent with the $r^{-2}$ distribution, being inferred from the quasi-static isothermal case, while those without IRAS sources tend to have less steep density gradient. (Fig. 1, Table 1)
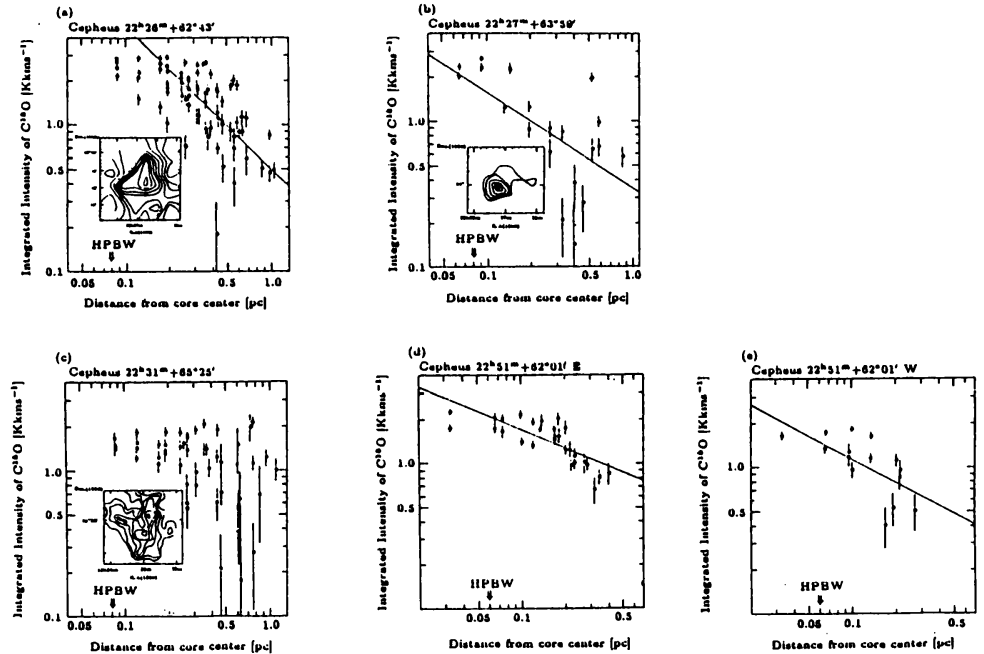

Fig. 1 - The $\mathrm{C}^{18} \mathrm{O}$ intensity, i.e. the gas column density, is plotted as a function of radius measured from the centers of the observed cores with associated IRAS sources ( $a$ and $b$ ) and without IRAS sources ( $c, d$, and $e$ ). The $C^{18} \mathrm{O}$ intensity for the cores with embedded IRAS sources ( $a$ and $b$ ) significantly decreases with the increasing radius from the core centers, while for the cores without IRAS sources (c, $d$, and $e$ ) the $C^{18} \mathrm{O}$ intensity remains relatively large even at large distances away from the centers. The straight lines show the result of least square fitting for the $C^{18} \mathrm{O}$ intensity. The map obtained with 4-m telescope at Nagoya university is inserted ( $a, b$, and $c)$.

E. Falgarone et al. (eds.), Fragmentation of Molecular Clouds and Star Formation, 500-501.

(c) 1991 IAU. Printed in the Netherlands. 
2. All the observed cores are stable in gravitational equilibrium. (Table 1)

3. The cores with steeper density gradient have larger average column density toward their centers. (Fig. 2)

These results suggest that cores with less steep density gradient contract in a quasistatic manner under gravitational equilibrium and evolve into cores with steeper density gradient to form protostars at their centers.

Table 1

\begin{tabular}{|c|c|c|c|c|c|c|c|c|}
\hline $\begin{array}{c}\text { [1] } \\
\text { Core Name }\end{array}$ & $\begin{array}{c}{[2\rfloor} \\
\tau\left(\mathrm{C}^{13} \mathrm{O}\right)\end{array}$ & $\begin{array}{l}{[3]} \\
T_{\text {.x }} \\
(K)\end{array}$ & $\begin{array}{r}{[4]} \\
\alpha\end{array}$ & $\begin{array}{l}{[5]} \\
\text { Size } \\
\text { (pc) }\end{array}$ & $\begin{array}{c}|6| \\
N\left(\mathrm{H}_{2}\right) \\
\left(\times 10^{21} \mathrm{~cm}^{-2}\right)\end{array}$ & $\begin{array}{c}{[7]} \\
\text { Volocity sradieat } \\
\left(\mathrm{kms}^{-1} \mathrm{pc}^{-1}\right)\end{array}$ & $\begin{array}{c}{[8]} \\
\text { Mass } \\
\left(M_{\odot}\right)\end{array}$ & $\begin{array}{c}{[9]} \\
\text { Virial Mase } \\
\left(M_{\odot}\right)\end{array}$ \\
\hline \multicolumn{9}{|l|}{ with an IRAS } \\
\hline \multicolumn{9}{|l|}{ point source } \\
\hline $22^{h} 26^{m}+62^{\circ} 43^{\prime}$ & 0.05 & 33 & -1.0 & 0.27 & 16.1 & 2.6 & 122 & 128 \\
\hline $22^{h} 27^{m}+63^{\circ} 59^{(1)}$ & 0.10 & 13 & -0.8 & 0.20 & 14.3 & 2.6 & 16 & 74 \\
\hline \multicolumn{9}{|l|}{ without an IRAS } \\
\hline \multicolumn{9}{|l|}{ point source } \\
\hline $22^{h} 31^{m}+65^{2} 25^{\prime}$ & 0.12 & 15 & $0^{(2)}$ & 0.40 & 10.5 & 1.6 & 83 & 109 \\
\hline $22^{h} 51^{m}+62^{\circ} 01^{\prime} E^{(l)}$ & 0.12 & 19 & -0.4 & 0.21 & 12.5 & - & 37 & 92 \\
\hline $22^{h} 51^{m}+62^{\circ} 01^{\prime} \mathrm{W}^{(1)}$ & 0.15 & 16 & .0 .5 & 0.15 & 10.0 & 3.0 & 11 & 56 \\
\hline
\end{tabular}

note) (1) These cores are associated with HII region.

(2) Correration coefficient is 0.3

Table 1 - We estimated the power law indices of the column density distribution from the least square fitting (column 4). This result suggests that the density profiles of the cores with IRAS sources are consistent with the $r^{-2}$ distribution under the assumption of spherical symmetry. On the contrary, cores without IRAS sources have less steep density gradient. The mass estimated from the observed column density (column 8 ) agrees well with the dynamical mass estimated from the observed line width (column 9), suggesting that these cores are in gravitational equilibrium.

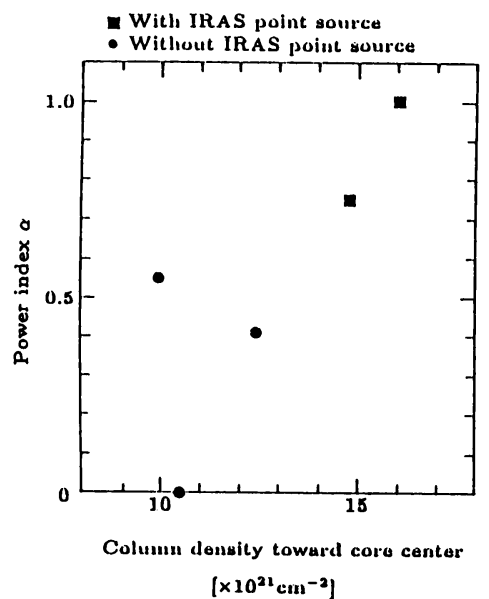

Fig. 2 - This figure shows the power law indices of the column density profiles versus the column density averaged over the radius of $0.15 \mathrm{pc}$ around the core centers. The cores with steeper density gradient have larger average colurnn density loward them. 